\title{
Dynamics of metastable vortex states in weakly pinned superconductors: A phenomenological model
}

\author{
G. Ravikumar \\ Technical Physics and Prototype Engineering Division, Bhabha Atomic \\ Research Centre, Mumbai-400085, India
}

October 29, 2018

\begin{abstract}
We present a phenomenological model for the vortex dynamics in the peak effect region of weakly pinned superconductors. We explain the history dependent dynamic response of the metastable vortex states subjected to a transport current and the hysteretic voltage-current characteristics observed in the vicinity of peak effect in weakly pinned superconductors. A strong variation in voltage current characteristics with the current sweep rate and the anomalous dependence of critical current density $J_{c}$ on the magnetic field sweep rate have also been accounted for by this model.
\end{abstract}

In weakly pinned superconductors, the competition between intervortex interaction and quenched disorder produces a sharp peak in the critical current density $J_{c}$ just below the normal state boundary. This phenomenon, known as the peak effect 1] occurs when the vortex lattice passes from a state with a high degree of spatial order to an amorphous pinned state [2, 3]. The peak effect is often accompanied by a marked history dependence in $J_{c}$ originating from different metastable configurations in which the vortex lattice could exist 田, 5, 6, 匇, 8].

Another aspect of the metastability associated with the peak effect is seen in the history dependent dynamic response of the vortex lattice to a transport current [6]. It is presumed that this behavior is due to the rearrangement of the vortex lattice from one metastable configuration to another 
aided by an external driving force [9]. Moreover, the process of rearrangement can be halted or in other words, a particular metastable configuration can be frozen in time, by merely switching off the transport current [9]. Switching on the current again after a time interval revives the further evolution of the metastable vortex configuration from where it was halted (long term "memory" effect). Further, the evolution of a metastable vortex configuration can be considerably slowed down by slightly lowering the transport current 9].

Additionally, Zhukov et al[10] have recently reported a magnetic field sweep rate $(d B / d t)$ dependent magnetization study in the peak region of $2 \mathrm{H}-\mathrm{NbSe}_{2}$ and in the fishtail region of $\mathrm{YBa}_{2} \mathrm{Cu}_{3} \mathrm{O}_{7}$. Their results imply that in the increasing field cycle $d J_{c} / d|d B / d t|<0$ at a fixed applied field. This so called "negative dynamic creep" phenomenon is in contast to the behavior expected from the thermally activated flux creep where $J_{c}$ is expected to increase logarithmically with the sweep rate $\left(d J_{c} / d|d B / d t|>0\right)$ 11. However, in the decreasing field cycle, it is found that $d J_{c} / d|d B / d t|>0$.

In transport experiments, $J_{c}$ is identified as the current density $J$ at which the electric field $E$ exceeds a threshold value. Usually pinning properties of the vortex lattice are uniquely characterized by the $J_{c}$ value and the corresponding $E-J$ relation. However, in the peak region of $2 \mathrm{H}-\mathrm{NbSe}_{2}$, $J_{c}$ is found to increase significantly with the rate at which $J$ is increased[9]. This amounts to the $J_{c}$ vs $T$ curve being much sharper when a large value of $d J / d t$ is used [9]. Moreover, $E-J$ curves themselves exhibit significant hysteresis in upward and downward current cycles 4 , 5, 6].

The history dependence of $J_{c}$ under different quasi-static field excursions was recently explained by a phenomenological model 12]. This model supposes that there exists a set of metastable vortex configurations, each corresponding to a different value of $J_{c}$. At a given field and temperature, different configurations differ only in the extent of lattice order. $J_{c}$ can thus be viewed as a macroscopic representation of a particular metastable vortex configuration. The most important assumption of this model is that there exists a stable vortex configuration with $J_{c}=J_{c}^{s t}$ which is unique for a given field and temperature. Depending on the particular magnetic history, a metastable configuration with $J_{c}$ either smaller or larger than $J_{c}^{s t}$ is produced. The extent of history dependence is assumed to be governed by the parameter $B_{r}$, which is a macroscopic measure of the metastability. Evolution of $J_{c}$ when the field changes from $B$ to $B+\delta B$ is postulated to be governed by 


$$
J_{c}(B+\delta B)=J_{c}(B)+\left(|\delta B| / B_{r}\right)\left[J_{c}^{s t}(B)-J_{c}(B)\right]
$$

Eq. 1 provides that a metastable configuration with $J_{c} \neq J_{c}^{s t}$ would evolve into the stable state when the field is repeatedly cycled by a small amplitude. Recently, the existence of such a stable state has been confirmed experimentally 13]. Physically, we can imagine that the process of repeated field cycling pumps the vortices in and out of the superconductor. In the absence of adequate thermal energy field cycling allows the vortex lattice to explore the configuration space and reorganize or anneal into the most stable vortex configuration. Thus the process of annealing is aided by the motion of vortices caused only by an external driving force such as a field change or a transport current larger than $J_{c}$ [9, 14.

We believe that the dynamic phenomenon mentioned above and the history dependence in critical currents are two different manifestations of the same phenomenon of metastability in the vortex state and therefore should be described by a common phenomenology. In this paper, we present a generalization of the phenomenological model described above, to account for (i) the history dependent dynamic response of the vortex lattice in the peak region (c.f Fig. 4 of Ref. [6]); (ii) the hysteresis in $E-J$ curves [4, 6], 6]; (iii) the dependence of $J_{c}$ on the current sweep rate used in $E-J$ measurements [9] and (iv) the so called negative dynamic creep phenomenon 10.

We postulate that, the time evolution of a metastable vortex configuration in terms of $J_{c}$ is described by the equation

$$
d J_{c} / d t=\left(J_{c}^{s t}-J_{c}\right) / \tau
$$

where $t$ is the time variable and $\tau$ is the time constant for the annealing process. We argue that the time constant $\tau$ is inversely related to the velocity $v$ of vortex motion, i.e., $\tau \sim|v|^{-1} \sim|E|^{-1}$ 14, 15. In other words, a larger velocity of vortices facilitates faster rearrangement of vortices into the stable configuration (annealing). The absolute value of $E$ (or $v$ ) implies that the process of rearrangement is independent of the direction of vortex motion.

Let us first examine the vortex rearrangement driven by a quasi-static field sweep, in the absence of transport current. If the field sweep rate is $d B / d t, E \sim d B / d t$ and the time constant $\tau\left(\sim|E|^{-1}\right)$ can be expressed as $\tau_{0} R /|d B / d t|$ where $R$ is a characteristic value of the sweep rate below which the field change can be considered quasi-static. The time constant $\tau_{0}(B, T)$ can be a function of the field $B$ and temperature $T$. Substituting the above expression for $\tau$ in Eq. 2, we get 


$$
d J_{c} / d B= \pm\left(J_{c}^{s t}-J_{c}\right) / B_{r}
$$

where $B_{r}=\tau_{0} R$ with upper (lower) sign for the field increasing (decreasing) case. Eq. 3 is the differential form of Eq. 1 describing the magnetic history dependent $J_{c}[12]$. Therefore, we conclude that the parameter $B_{r}$ which is a measure of the history dependence in $J_{c}$, is directly related to the time constant $\tau_{0}$ which, as would be shown below, governs the dynamics of metastable vortex states subjected to an external driving force.

We now consider the dependence of $J_{c}$ on magnetic field sweep rate for a fixed applied field. When the external field $B(t)$ increases (or decreases) linearly in time, we can write $t= \pm\left[B(t)-B_{i}\right] / \dot{B}$. The upper (lower) sign is again applicable for increasing (decreasing) field. $B_{i}$ is some initial field where the field sweep started and $\dot{B}$ (positive) is the magnitude of the sweep rate $d B / d t$. Substituting for $t$ in Eq. 2, we get

$$
d J_{c} / d \dot{B}=\mp\left[\left(B(t)-B_{i}\right) / \dot{B}^{2}\right]\left(J_{c}^{s t}-J_{c}\right) / \tau
$$

In the increasing field cycle (upper sign) $\left(B(t)-B_{i}\right)$ is positive and $J_{c}<J_{c}^{s t}$ in the peak region [12, 13. Therefore $d J_{c} / d \dot{B}<0$ (negative dynamic creep) as observed in experiments 10]. On the other hand, in the decreasing field cycle (lower sign) $d J_{c} / d \dot{B}>0$ because $J_{c}>J_{c}^{s t}$ (see Ref. [12]) and $\left(B(t)-B_{i}\right)$ is negative. In other words, the negative dynamic creep observed in the increasing field cycle [10] is a manifestation of the finite time delay needed for the vortex lattice to become more disordered.

We now focus on the transport current driven annealing process governed by Eq. 2 where the time constant $\tau \sim|E(J)|^{-1}$. For $E(J)$, we choose the simple form $E(J) \sim\left(J / J_{c}-1\right)$ for $J>J_{c}$ and thus

$$
\tau(J)=\tau_{0} /\left(J / J_{c}-1\right)
$$

For $J<J_{c}, E(J)=0$ and therefore $\tau \rightarrow \infty$. The specific form chosen for $E(J)$ is however not critical for the results we are going to present. Any other form which makes $\tau \rightarrow \infty$ as $J \rightarrow J_{c}$ would equally serve to illustrate the results in this paper.

Eq. 2 and Eq. 5 suggest that irrespective of whether (i) $J_{c}<J_{c}^{s t}$ or (ii) $J_{c}>J_{c}^{s t}$, a transport current $J>J_{c}$ causes $J_{c}$ to increase or decrease respectively towards the stable value. In other words, while $J>J_{c}, J_{c}$ and thereby $\tau$ change with time, resulting in the time dependence of the voltage response through the relation $E(J) \sim\left(J / J_{c}(t)-1\right)$, even when the transport current $J$ is kept fixed. 
Combining Eq. 2 and Eq. 5 and substituting the dimensionless quantities $j_{c}(t)=J_{c}(t) / J_{c}^{s t}, j=J / J_{c}^{s t}$ and $t \rightarrow t / \tau_{0}$, a formal solution for $j_{c}(t)$ can be written as

$$
j_{c}(t)=1+\left[j_{c}\left(t_{0}\right)-1\right] \exp \left[-\int_{t_{0}}^{t} d t\left(j / j_{c}(t)-1\right)\right]
$$

where $j_{c}\left(t_{0}\right)$ is the normalized critical current of the vortex configuration in which the system is initially prepared at time $t_{0}$. Here we have two cases of interest viz., (i) $j_{c}\left(t_{0}\right)<1$ and (ii) $j_{c}\left(t_{0}\right)>1$ which are henceforth referred to as superheated and supercooled vortex configurations respectively 13. The superheated configuration can be achieved by entering the peak region either by increasing the field at constant temperature [12, 13] or by increasing temperature at a constant field. On the other hand, the supercooled state is achieved by either decreasing the field from above the normal state boundary while keeping the temperature constant [8, 12, 13] or by cooling the superconductor in a field [7, 8].

We solve Eq. 6 iteratively to obtain the time evolution of $j_{c}(t)$ in response to a step increase in the transport current density $j$ at $t=t_{0}$. In Fig. 1 , we present the time dependence of $j_{c}(t)$ and $E(t) \sim\left(j / j_{c}(t)-1\right)$ for the superheated vortex configuration, i.e, $j_{c}\left(t_{0}\right)<1$. The voltage response decreases with time as observed experimentally (c.f. Fig. 4(b) of Ref. [6] and Fig. 2 of Ref. [9]). In the inset of Fig. 1(b), we show $E(t)$ vs $\ln (t)$ at different driving currents, which is in excellent qualitative agreement with the experimental results (c.f. Ref. 90). In Fig. 2, we present $j_{c}(t)$ and $E(t)$ obtained by starting from a supercooled vortex configuration (i.e., $j_{c}\left(t_{0}\right)>1$ ), which is again in good qualitative agreement with experiments(c. f. Fig. 4(b) of Ref. [6]).

Let us now consider the situation in Fig. 1, but with the driving current $j$ switched off to zero during the interval $t_{1}<t<t_{2}$. At time $t_{1}$, the vortex lattice is frozen into a metastable configuration corresponding to $j_{c}=j_{c}\left(t_{1}\right)$ as the time constant $\tau \rightarrow \infty$ for $j<j_{c}$. When the current is switched on again at $t=t_{2}, j_{c}$ begins to evolve from the value $j_{c}\left(t_{1}\right)$ according to Eq. 6 as shown in Fig. 3(a). Similarly, one can consider the case of reducing the current $j$ by a small amount over a time interval as shown in Fig. 3(b). This merely slows down the process of evolution of $J_{c}$ towards a stable value $J_{c}^{s t}$. The calculated voltage responses shown in Figs. 3(a) and 3(b) compare very well with the results of Ref. [9].

The above discussion provides a framework for the annealing process mediated by a field change 12, 13]. When the critical state is set up, shielding 
currents (just like eddy currents) are set up at a high current density $(>>$ $\left.J_{c}\right)$ [16] which decay to the value $J_{c}$ with a characteristic time constant $T \approx$ $\mu_{0} a^{2} / 4 \rho_{f f}$ governed by the flux flow resistance $\rho_{f f}=\rho_{n} H / H_{c 2}$, where $\rho_{n}$ is the normal state resistivity, $H$ and $H_{c 2}$ are the external field and the upper critical field respectively, $\mu_{0}=4 \pi \times 10^{-7} \mathrm{~W} / \mathrm{A} . \mathrm{m}$ and $a$ is the sample dimension. This is equivalent to a pulse of transport current $J_{\text {mean }}>J_{c}$ of duration $T$ which causes a partial annealing of the metastable vortex state.

This model can also be used for calculating the $E-j$ relations. We consider that the transport current density $j\left(>j_{c}\right)$ is increased in small steps of $\Delta j$ each of duration $\Delta t$. We calculate the $j_{c}$ and the corresponding $E(j)$ values at the end of each current step. In Fig. 4 we plot the $E-j$ curves obtained with two different values of the current sweep rate $d j / d t$ $(\simeq \Delta j / \Delta t)$. Clearly, the critical current density inferred from these curves would be smaller when $d j / d t$ is larger, just as observed in experimental studies(c.f. Fig. 1 of Ref. [9]). Further, the shape of $E-j$ curves differ dramatically with the current sweep rate.

We also studied the $E-j$ curves in both upward and downward current cycles with a fixed $d j / d t$. In Fig. 5(a) we consider the case where the vortex lattice is iniatially prepared in a metastable configuration with $j_{c}\left(t_{0}\right)<1$ (superheated state) and in Fig. $4(\mathrm{~b})$, for the case $j_{c}\left(t_{0}\right)>1$ (supercooled state). The calculation clearly brings out the hysteretic $E-J$ behavior usually seen in the peak region and the results compare remarkably well

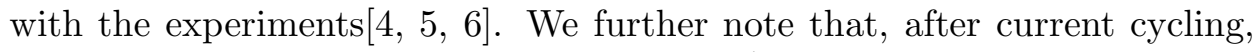
$j_{c}\left(J_{c}\right)$ always settles to the value $1\left(J_{c}=J_{c}^{s t}\right)$ as shown in Ref. [5].

The results presented here are contrary to the notion that the $E-J$ relation and the $J_{c}$ value inferred from it, uniquely characterize the pinning properties of the underlying vortex structure in all circumstances. We argued instead that while the transport current $J$ is higher than the threshold value $J_{c}$, the $J_{c}$ value itself changes irreversibly, leading to the hysteresis in the $E-J$ relations.

We caution here that the simple model presented here explains a variety of dynamic response of the vortex lattice to a transport current above the macroscopic threshold value $J_{c}$. However, it can not account for the $a c$ dynamic response for current values below $J_{c}$ 17]. A qualitative mechanism for the $a c$ dynamic response was recently presented by Paltiel et al[14] by considering the disordered vortex phase, injected into the superconducting sample across the surface imperfections, coexisting with the ordered phase in the interior of the sample. However, an explicit connection between this mechanism and the model developed here is not yet available. 
In conclusion, we have presented a macroscopic model which accounts for the dynamics of the vortex lattice in the peak effect region. We explained the history dependent dynamic response of the metastable vortex states subjected to a transport current and the hysteretic voltage-current characteristics observed in the peak region of weakly pinned superconductors such as $2 \mathrm{H}-\mathrm{NbSe}_{2}$. We are also able to explain the strong variation in voltage current characteristics with the current sweep rate. Further, the so called phenomenon of "negative dynamic creep", viz., the anomalous dependence of $J_{c}$ on the magnetic field sweep rate in the peak region is also explained.

The author thanks Prof. S. Bhattacharya, Dr. K. V. Bhagwat, Prof. A. K. Grover, Prof. S. Ramakrishnan and Dr. V. C. Sahni for discussions and critical reading of the manuscript.

\section{REFERENCES}

1. M. J. Higgins and S. Bhattacharya, Physica C 257, 232 (1996) and references therein.

2. A. I. Larkin and Y. N. Ovchinnikov, Zh. Eksp. Teor. Fiz 65, 1704 (1973) [Sov. Phys. JETP 38, 854 (1974)].

3. P. L. Gammel et al, Phys. Rev. Lett. 80, 833 (1998).

4. M. Steingart, A. G. Putz and E. J. Kramer, J. Appl. Phys. 44, 5580 (1973).

5. R. Wordenweber, P. H. Kes and C. C. Tsuei, Phys. Rev. B 33, 3172 (1986).

6. W. Henderson, E. Y. Andrei, M. J. Higgins and S. Bhattacharya, Phys. Rev. Lett. 77, 2077 (1996).

7. G. Ravikumar et al, Phys. Rev. B 57, R11069 (1998).

8. G. Ravikumar et al, Phys. Rev. B 61, 12490 (2000).

9. Z. L. Xiao, E. Y. Andrei and M. J. Higgins, Phys. Rev. Lett. 83, 1664 (1999).

10. A. A. Zhukov et al, Phys. Rev. B 61, R886 (2000).

11. L. Pust, Supercond. Sci. Technol. 3, 598 (1990). 
12. G. Ravikumar et al., Phys. Rev. B 61, R6479 (2000).

13. G. Ravikumar et al., Submitted to Phys. Rev. B.

14. Y. Paltiel et al, Nature (London) 403, 398 (2000).

15. The time constant $\tau$ should be related to the transit time of the vortices across the sample and is thus inversely related to the velocity of vortex motion (S. Bhattacharya, private communication).

16. M. N. Wilson, Superconducting Magnets, Oxford:Clarendon Press, (1983).

17. W. Henderson, E. Y. Andrei and M. J. Higgins, Phys. Rev. Lett. 81, 2352 (1998).

\section{FIGURE CAPTIONS}

Fig. 1: (a) Time evolution of $j_{c}(t)$ in response to a step change in the transport current $j$ (dotted line). Initial vortex state corresponds to a superheated vortex configuration with $j_{c}\left(t_{0}=0\right)=0.5(<1)$. The corresponding voltage response is shown in (b). In the inset of (b), this response is plotted as $E$ vs $\ln (t)$ for different drive currents (compare with Fig. 2 of Ref. [9]).

Fig. 2: Time dependence of $j_{c}(t)$ and $E(t)$ in response to a step current pulse (dotted line), The initial vortex state corresponds to a supercooled vortex configuration with $j_{c}\left(t_{0}=0\right)=1.5(>1$.

Fig. 3: (a) Time dependent response of the superheated state considered in Fig. 1, but with the drive current $j$ switched off during the interval $t_{1}<t<t_{2}$. Note that $E\left(t=t_{2}\right)=E\left(t=t_{1}\right)$ which is referred to as the "long term memory" effect (see text). (b) same as (a) but the current $j$ (dotted line) is only lowered by a small amount $\left(j>j_{c}(t)\right)$. Note that the time dependence of $E(t)$ is significantly slowed down.

Fig. 4: Calculated $E-j$ relations for two different values of current sweep rate $d j / d t$ as indicated. $j_{c}^{i}=0.5$ is the critical current of the vortex state in which the superconductor is initially prepared.

Fig. 5: Calculated $E-j$ relation for upward and downward current cycles as indicated by the arrows. In (a) vortex state is initially prepared with a critical current density $j_{c}^{i}<1$ and in (b) initial state 
corresponds to $j_{c}^{i}>1$. The $d j / d t$ is kept fixed at 0.1 . In both cases $E(j)$ becomes zero at $j=1\left(J_{c}=J_{c}^{s t}\right)$ in the downward current cycle. 


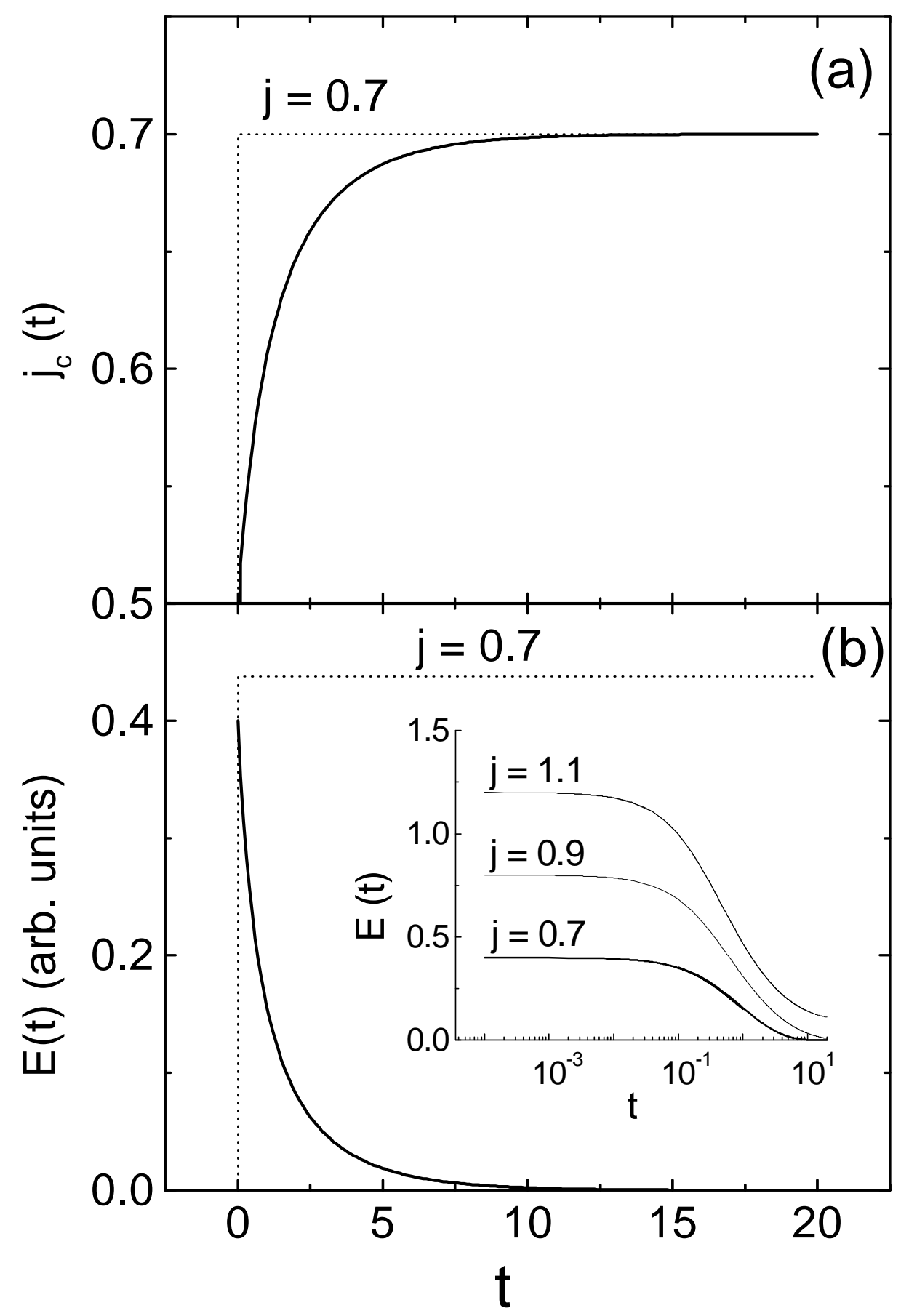

Fig. 1 - Ravikumar 


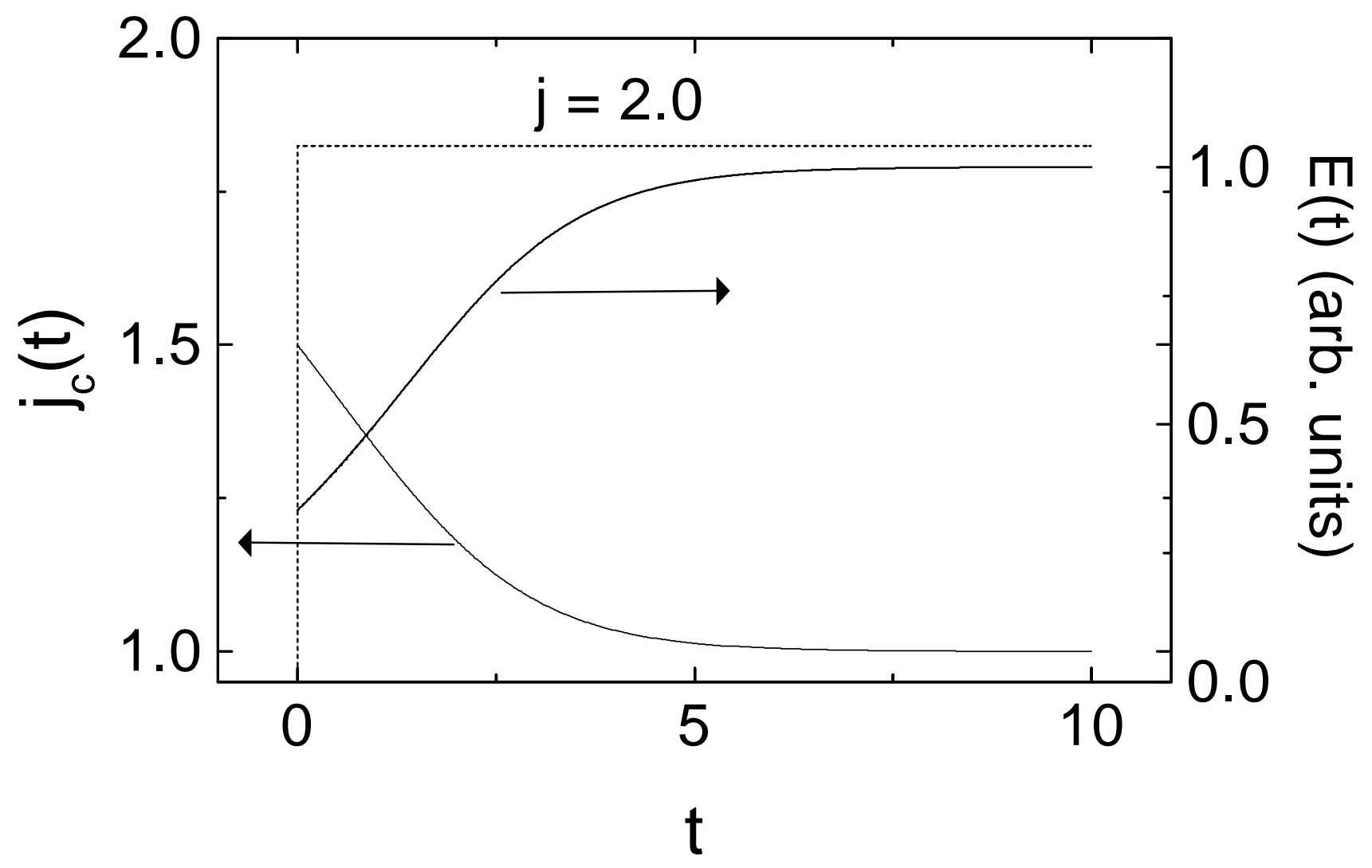

Fig.2 - Ravikumar 

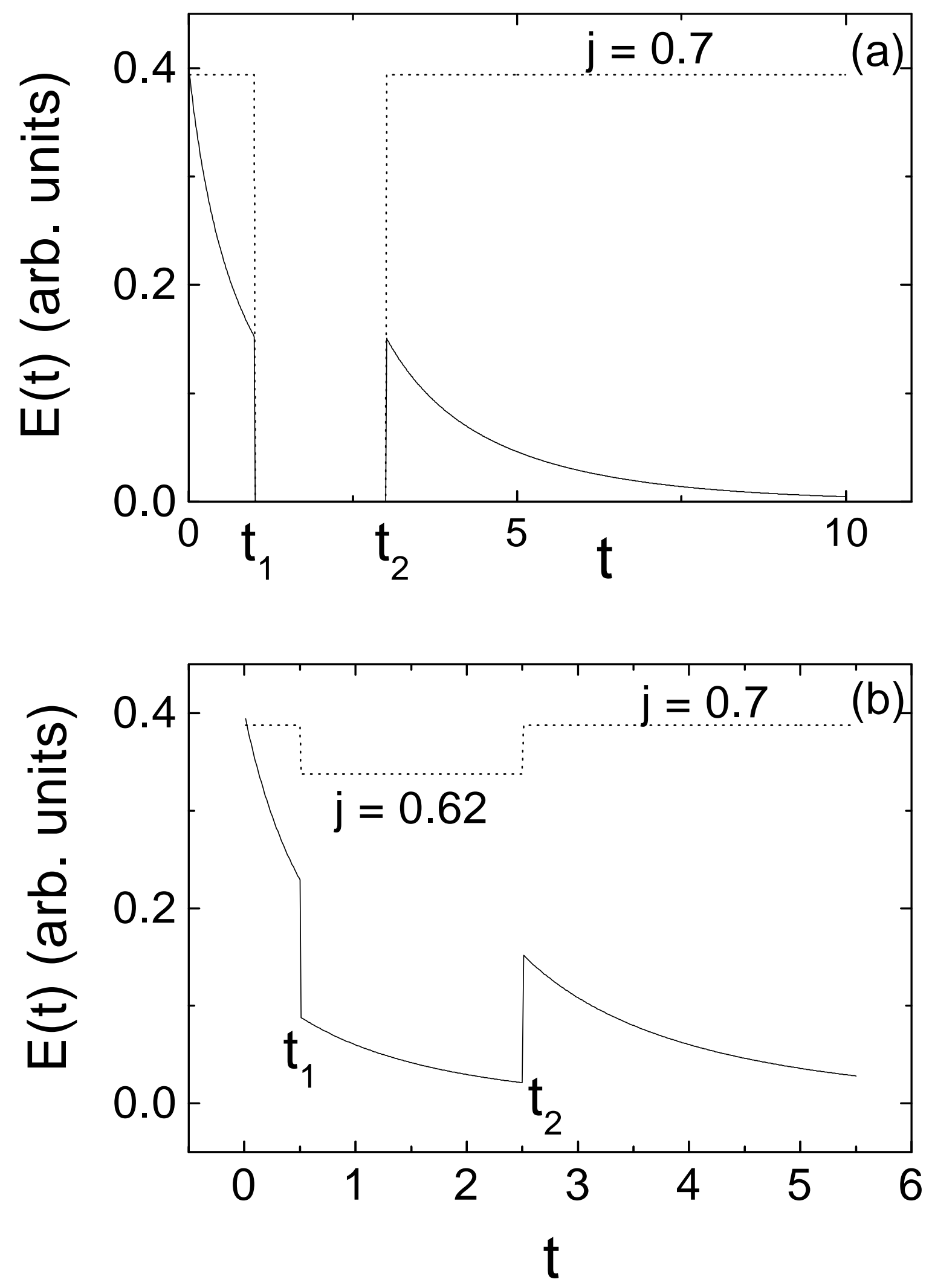

Fig. 3 - Ravikumar 


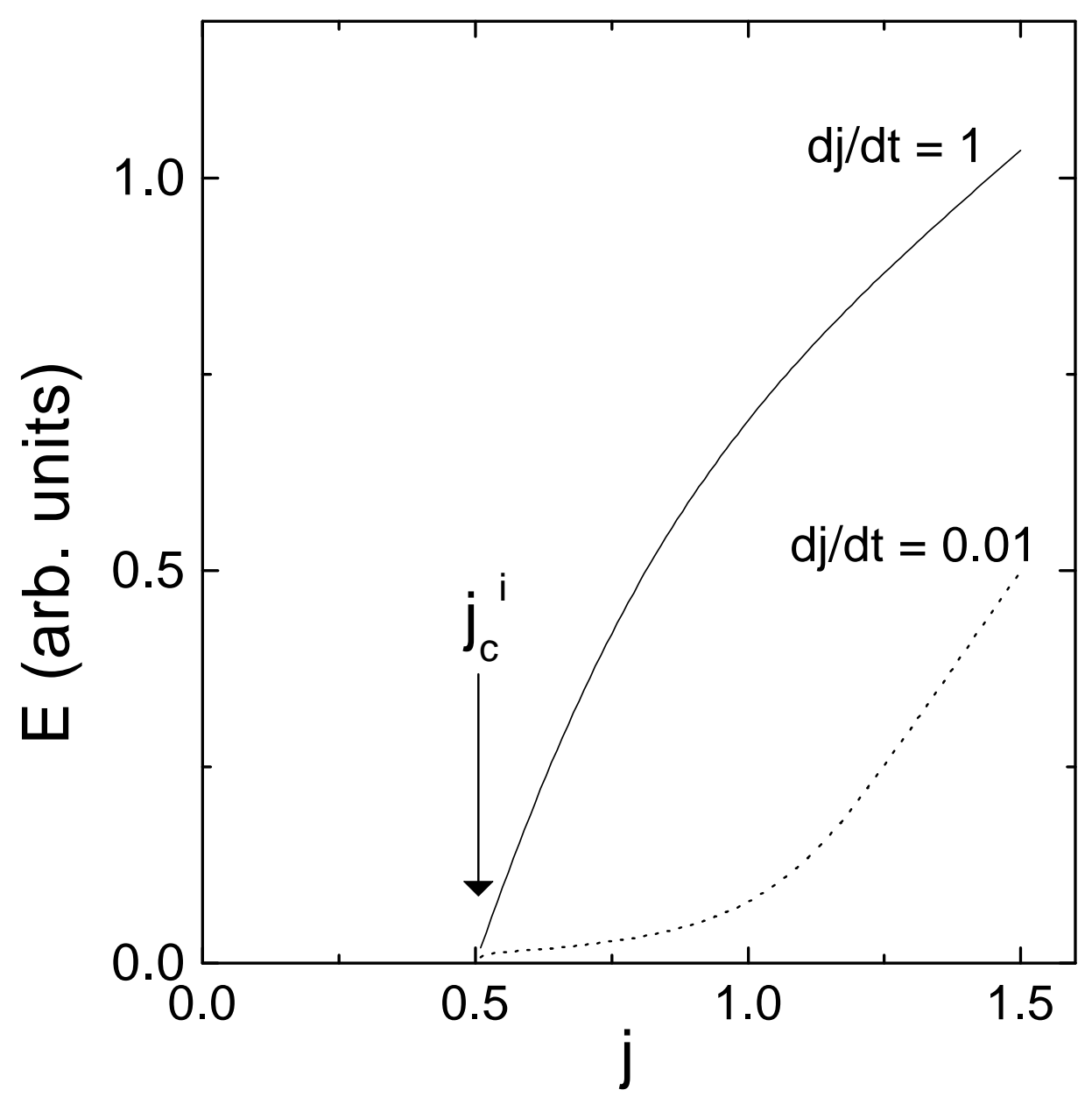

Fig. 4 - Ravikumar 


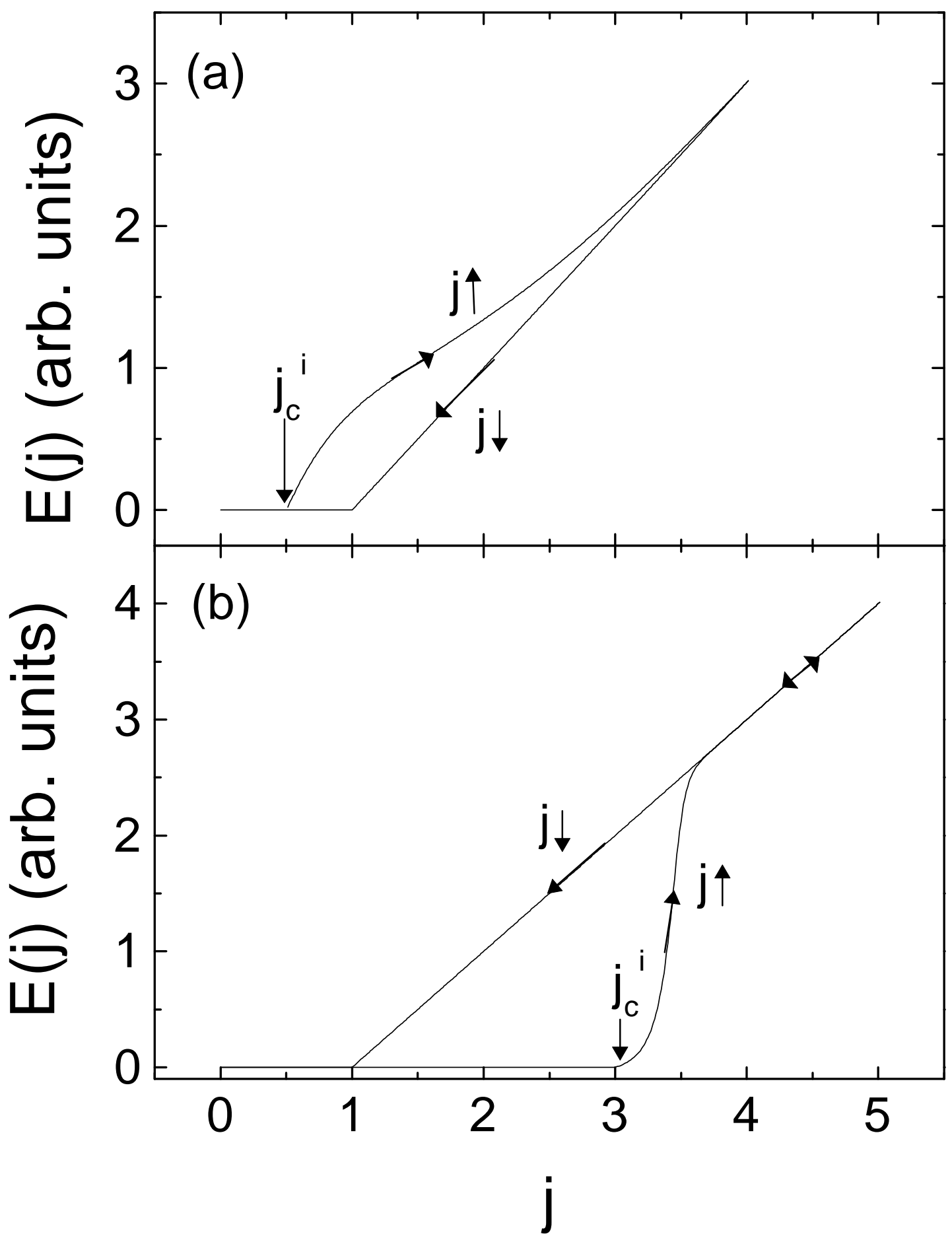

Fig. 5 - Ravikumar 